\title{
COACTIONS OF HOPF ALGEBRAS ON CUNTZ ALGEBRAS AND THEIR FIXED POINT ALGEBRAS
}

\author{
ANNA PAOLUCCI
}

(Communicated by Palle E. T. Jorgensen)

\begin{abstract}
We study coactions of Hopf algebras coming from compact quantum groups on the Cuntz algebra. These coactions are the natural generalization to the coalgebra setting of the canonical representation of the unitary matrix group $U(d)$ as automorphisms of the Cuntz algebra $O_{d}$.

In particular we study the fixed point subalgebra under the coaction of the quantum compact groups $U_{q}(d)$ on the Cuntz algebra $O_{d}$ by extending to any dimension $d<\infty$ a result of Konishi (1992).

Furthermore we give a description of the fixed point subalgebra under the coaction of $S U_{q}(d)$ on $O_{d}$ in terms of generators.
\end{abstract}

\section{INTRODUCTION}

Model actions of finite-dimensional Hopf algebras on Cuntz algebra have been investigated in [2] and [7]. In these notes we take a first step in the study of model actions $\Gamma$ of Hopf algebras coming from compact matrix quantum groups $G$ on a Cuntz algebra and their fixed point algebras. We consider an analogue for coalgebras of the classical action of the unitary matrix group on a Cuntz algebra. The fixed point subalgebra of the Cuntz algebra under this natural coaction of the quantum $U_{q}(d)$ is considered. The case $d=2$ was already found in [9]. We extend it to the general case of any $d<\infty$ and characterize the fixed point subalgebras of $O_{d}$ under the coaction of respectively $U_{q}(d)$ denoted by $\left(O_{d}\right)^{U_{q}(d)}$. This algebra carries the canonical endomorphism $\sigma_{U_{q}(d)}$ which is the restriction of the canonical endomorphism $\hat{\sigma}$ of $O_{d}$. We characterize $\left(O_{d}\right)^{S U_{q}(d)}$ as fixed point subalgebras of $O_{d}$ under the coactions of $U_{q}(d)$ and $S U_{q}(d)$ respectively.

\section{BASIC DEFINITIONS}

Let $B_{\infty}$ be the infinite braid group. One of the usual models for $B_{n}$ is as follows. Fix on each of two parallel lines in 3-space, an "upper" and a "lower" one, $n$ points labelled by the numbers $1,2, \ldots, n$. We require that this labelling respect the natural ordering of our index set. A braid is obtained by connecting each of the upper points with a point of the lower line by a curve going only downward. Multiplication of two braids is defined by connecting the lower points of the first braid with the upper points of the second braid with matching labels. Similarly

Received by the editors August 4, 1995.

1991 Mathematics Subject Classification. Primary 46M05, 16W30, 81R50.

Key words and phrases. $C^{*}$-algebras, Hilbert spaces, representation, corepresentation, duality.

(C)1997 American Mathematical Society 
$B_{\infty}$ can be realized by countably many points such that all but finitely many of them go straight downwards. Hence we see that we can realize $B_{\infty}$ as an inductive limit of the groups $B_{n}$, by the inclusion maps

$$
B_{n} \subset B_{n+1} \text { which sends } g_{n} \rightarrow g_{n} \otimes 1
$$

where by the tensor we mean to add an extra label on the "upper" and "lower" line connected by a straight line and $B_{n}$ will be the subgroup of $B_{\infty}$ generated by $g_{1}, \ldots, g_{n}$. The shift $\sigma: B_{n} \rightarrow B_{n+1}$ which sends $g_{n} \mapsto 1 \otimes g_{n}=\sigma\left(g_{n}\right)$, by the compatibility with the inclusion then extends to an endomorphism which we denote by the same letter $\sigma: B_{\infty} \rightarrow B_{\infty}$.

Cuntz introduced his $C^{*}$-algebra $O_{d}$ in [1] and showed that isometries $S_{i}$, for $i=1, \ldots, d$ satisfying

$$
S_{i}^{*} S_{j}=\delta_{i j} 1, \quad \sum_{i=1}^{d} S_{i} S_{i}^{*}=1
$$

generate a unique $C^{*}$-algebra $O_{d}$. The ${ }^{*}$-algebra over the complex numbers with unit 1 generated by the $S_{i}$ with the above relations will be denoted by ${ }^{\circ} O_{d}$, the algebraic part of $O_{d}$. The linear span of the $S_{i}$ 's for $i=1, \ldots, d$ will be denoted by $H$ and it is the canonical Hilbert space. The scalar product on $H$ is defined by $\left\langle S, S^{\prime}\right\rangle 1=S^{*} S^{\prime}$. The linear subspace generated by $S_{i_{1}} \cdots S_{i_{r}}$ will be denoted by $H^{r}$, and the linear span of terms of the form $S_{i_{1}} \cdots S_{i_{r}} S_{j_{s}}^{*} \cdots S_{j_{1}}^{*}$ by $\left(H^{s}, H^{r}\right)$, where by $\left(H^{s}, H^{r}\right)$ we mean the set of intertwiners from $H^{s}$ to $H^{r}, S_{i} \in(\mathbf{C}, H)$ is an intertwiner from $\mathbf{C}$ to $H$. We consider $H$ a Hilbert space of dimension $d<\infty$.

Let $G$ be a compact quantum group which we denote with the pair $(A, \Delta)$ where $A$ is a $C^{*}$-algebra and $\Delta$ is the comultiplication as in [14]. Let $R$ be the operator from the two-fold tensor product of $H$ into itself. We say that $R$ satisfies the Yang-Baxter equations if the following equation holds:

$$
R_{12} R_{13} R_{23}=R_{23} R_{13} R_{12}
$$

where the lower indices mean the position of $R$ in the 3-tensor. We denote by $c_{H, H}=\tau(R)$ the composition of $R$ with the flip $\tau$. Then $c_{H, H} \in\left(H^{2}, H^{2}\right)$. It gives an element $g_{0}=c_{H, H}$ of the braid group $B_{\infty}$ and by using the shift $\sigma$, we can write any generator of $B_{\infty}$ in terms of $g_{0}$ and $\sigma$ as follows. Let $g_{0}=c_{H, H}$, $g_{1}=\sigma\left(g_{0}\right)=1 \otimes g_{0}, g_{i}=\sigma^{(i)}\left(g_{0}\right)$. Set $\theta\left(g_{i}\right)=c_{H^{i}, H^{i}} \in\left(H^{i}, H^{i}\right)$ for every $i=1, \ldots, n-1$ and similarly we define $c_{H^{r}, H^{s}}$ for any $r, s$, by the equation

$$
\begin{gathered}
c_{H^{r}, H^{s}}=\prod_{s-1} \prod_{s-2} \cdots \prod_{r} \\
\prod_{s-1}=\left(\tau_{H^{r}, H^{r}}(R) \circ \tau_{H^{r+1}, H^{r+1}}(R) \circ \cdots \circ \tau_{H^{s-1}, H^{s-1}}(R)\right), \\
\prod_{s-2}=\left(\tau_{H^{r}, H^{r}}(R) \circ \cdots \circ \tau_{H^{s-2}, H^{s-2}}(R)\right), \ldots, \prod_{r}=\tau_{H^{r}, H^{r}}(R)
\end{gathered}
$$

where $\tau_{H^{r}, H^{s}}(R)$ stands for a copy of $R$, after the flipping in the $r, r+1$ position. The image of the operator $\theta\left(g_{0}\right)$ in terms of the generators of $O_{d}$ have the following form:

$$
\theta\left(g_{0}\right)=\sum_{i_{1}, i_{2}} c_{H, H}\left(S_{i_{1}} \otimes S_{i_{2}}\right) S_{i_{p(2)}}^{*} \otimes S_{i_{p(1)}}^{*}, \quad g_{0} \in B_{\infty}
$$


where we then identify $S_{i_{1}} \otimes S_{i_{2}}$ with $S_{i_{1}} S_{i_{2}}$ and $p$ stands for the unique permutation of $(1,2)$ associated to the element $g_{0} \in B_{\infty}$ which in turn implies $g_{0} \in B_{n}$, for some $n$.

In general given $g \in B_{n}$, the map $g \mapsto p(g)$ induces a surjective morphism of groups from the braid group $B_{n}$ onto the symmetric group $S(n)$, hence the form of the operator $\theta$ for any $g \in B_{n}$ will be:

$$
\theta(g)=\sum_{i_{1} \cdots i_{k}} c_{i_{1} \cdots i_{n}}^{p\left(i_{n}\right), \ldots, p\left(i_{1}\right)} S_{i_{1}} \cdots S_{i_{n}} S_{p\left(i_{n}\right)}^{*} \cdots S_{p\left(i_{1}\right)}^{*}
$$

where we denote by $c_{i_{1} \ldots i_{k}}^{p\left(i_{k}\right), \ldots, p\left(i_{1}\right)}$ the matrix elements of the operator $c_{H^{n}, H^{n}}$ as defined before.

\section{Coactions of COMPaCt Quantum groups on Cuntz algebras}

Let $(A, \Delta)$ be a compact quantum group. Let $u \in M_{n} \otimes A$ be a unitary corepresentation of $A$. This means that $u$ is unitary and that $(i \otimes \Delta) u=u_{12} u_{13}$. We write $u=\sum e_{i j} \otimes u_{i j}$. Then $u$ unitary means

$$
1=u^{*} u=\left(\sum e_{i j}^{*} \otimes u_{i j}^{*}\right)\left(\sum e_{p q} \otimes u_{p q}\right)=\sum_{i, q, p} e_{j q} \otimes u_{p j}^{*} u_{p q}
$$

So $\sum_{p} u_{p r}^{*} u_{p q}=\delta_{r q} 1$. Similarly $1=u u^{*}$ will give $\sum_{p} u_{r p} u_{q p}^{*}=\delta_{r q} 1$. The fact that $(1 \otimes \Delta) u=u_{12} u_{13}$ means

$$
\sum e_{i j} \otimes \Delta\left(u_{i j}\right)=\sum e_{i j} e_{p q} \otimes u_{i j} \otimes u_{p q}=\sum e_{i q} \otimes u_{i p} \otimes u_{p q} .
$$

Thus $\Delta\left(u_{i j}\right)=\sum_{p} u_{i p} \otimes u_{p j}$. Observe that the classical compact group $U(d)$ acts on the Cuntz algebra $O_{d}$ as an automorphism group. When dealing with coalgebras in the quantum group setting, the following coaction is the natural choice. Consider the elements $T_{i}=\sum S_{j} \otimes u_{j i} \in O_{d} \otimes A$. We have

$$
T_{i}^{*} T_{j}=\left(\sum S_{p}^{*} \otimes u_{p i}^{*}\right)\left(\sum S_{q} \otimes u_{q j}\right)=\sum S_{p}^{*} S_{q} \otimes u_{p i}^{*} u_{q j}=\sum 1 \otimes u_{p i}^{*} u_{p j}=\delta_{i j} 1
$$

and

$$
\sum T_{i} T_{i}^{*}=\left(\sum S_{p} \otimes u_{p i}\right)\left(\sum S_{q}^{*} \otimes u_{q i}^{*}\right)=\sum S_{p} S_{q}^{*} \otimes u_{p i} u_{q i}^{*}=\sum S_{p} S_{q}^{*} \otimes 1=1 .
$$

Thus there exists a $*$-homomorphism $\Gamma: O_{d} \rightarrow O_{d} \otimes A$ defined by $\Gamma\left(S_{i}\right)=\sum_{p} S_{p} \otimes$ $u_{p i}$. Then

Definition 1. Let $B$ be a $C^{*}$-algebra and $\pi$ be a $*$-homomorphism from $B$ to $B \otimes A$. We say that $\pi$ is a coaction of a compact quantum group $G=(A, \Delta)$ on $B$ if

$$
\left(\pi \otimes \operatorname{id}_{A}\right) \pi=(\mathrm{id} \otimes \Delta) \pi
$$

where $\Delta$ is the comultiplication. This is equivalent to saying that the following diagram commutes:

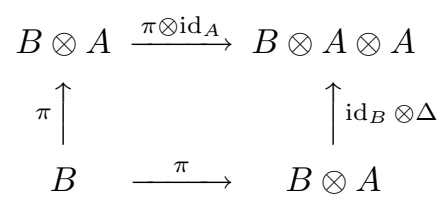


Then, the $*$-homomorphism $\Gamma$ defined before is a coaction. In fact we have $(i \otimes \Delta) \Gamma\left(S_{p}\right)=\sum S_{p} \otimes u_{q r} \otimes u_{r p}$ and $(\Gamma \otimes i) \Gamma\left(S_{p}\right)=\sum S_{p} \otimes u_{q r} \otimes u_{r p}$. It follows that $(i \otimes \Delta) \Gamma=(\Gamma \otimes i) \Gamma$. Thus $\Gamma$ is a coaction of $A$ on $O_{d}$. We have the following result $[9]$.

Theorem 2. For a given unitary corepresentation $w \in M_{d} \otimes A$ of a compact quantum group $G=(A, \Delta)$ there is a coaction $\Gamma$ determined by $\Gamma\left(S_{i}\right)=\sum_{j=1}^{d} S_{j} \otimes w_{j i}$ for any $1 \leq i \leq d$.

We now want to describe the fixed point subalgebra under this coaction $\Gamma$. It is an analogue to the fixed point subalgebra for the $U(d)$ group action in $O_{d}$.

Definition 3. Let $B$ be a $C^{*}$-algebra and let $\pi$ be a coaction of $G$ on $B$. We define the fixed point subalgebra $B^{\pi}$ of $B$ by $\pi$ as

$$
B^{\pi}=\left\{x \in B: \pi(x)=x \otimes 1_{A}\right\} .
$$

Denote by $M_{d}^{k}$ the $k$-times tensor product of the $d \times d$ matrix algebra $M_{d}$ and define a canonical embedding $\eta: M_{d}^{k} \rightarrow O_{d}$ by

$$
\eta\left(e_{i_{1} j_{1}} \otimes \cdots \otimes e_{i_{k} j_{k}}\right)=S_{i_{1}} \cdots S_{i_{k}} S_{j_{k}}^{*} \cdots S_{j_{1}}^{*}
$$

where $\left\{e_{i j}\right\}_{i, j=1}^{d}$ is a system of matrix units of $M_{d}$. This embedding is compatible with the canonical inclusion of $M_{d}^{k}$ into $M_{d}^{k+1}$. We denote by $M_{d}^{\infty}$ the UHF algebra which is the inductive limit of $\left\{M_{d}^{k}\right\}_{k=1}^{\infty}$. Observe that the UHF algebra $M_{d}^{\infty}$ can be considered as a $C^{*}$-subalgebra of $O_{d}$ through the embedding $\eta$. Define $w^{k}=w \otimes \cdots \otimes w$ to be the tensor product of the unitary corepresentation $w$ with itself $k$ times. Clearly $w^{k}$ is a unitary corepresentation if $w$ is. The restriction of the coaction $\Gamma$ to the UHF algebra $M_{d}^{\infty}$ is also a coaction of the compact quantum group $G$ on $M_{d}^{\infty}$. Set $\phi=\left.\Gamma\right|_{U H F}$. Then it satisfies

$$
\begin{aligned}
& \phi\left(e_{i_{1} j_{1}} \otimes \cdots \otimes e_{i_{k} j_{k}}\right) \\
& \quad=\sum_{a_{1}, \ldots, a_{k}, b_{1}, \ldots, b_{k}} e_{a_{1} b_{1}} \otimes \cdots \otimes e_{a_{k} b_{k}} \otimes w_{a_{1} i_{1}} \cdots w_{a_{k} i_{k}} w_{a_{k} j_{k}}^{*} \cdots w_{a_{1} j_{1}}^{*},
\end{aligned}
$$

for every $k$ a positive integer. Therefore $\phi$ can be represented in the form $\phi(x)=$ $w^{k}\left(x \otimes 1_{A}\right)\left(w^{k}\right)^{*}$, for every $x \in M_{d}^{k}$. In [9] $\phi$ of this form is called a product type coaction of the compact quantum group $G=(A, \Delta)$ on the UHF algebra $M_{d}^{\infty}$. Let us turn now to the fixed point subalgebra related to the coaction $\Gamma$ of a matrix quantum group on $O_{d}$. Clearly the fixed point subalgebra will depend on the unitary corepresentation $u$.

Lemma 4. The canonical endomorphism $\sigma$ of the Cuntz algebra $O_{d}$ leaves the fixed point subalgebra in $O_{d}$ invariant under $\Gamma$.

Proof. Let $\sigma(x)=\sum_{i=1}^{d} S_{i} x S_{i}^{*}$, and let $x$ be a fixed point so that $\Gamma(x)=x \otimes 1$. Then

$$
\begin{aligned}
\Gamma(\sigma(x)) & =\sum_{i=1}^{d} \Gamma\left(S_{i} x S_{i}^{*}\right)=\sum_{i=1}^{d} \Gamma\left(S_{i}\right)(x \otimes 1) \Gamma\left(S_{i}^{*}\right) \\
& =\sum_{i, p, q}\left(S_{p} \otimes u_{p i}\right)(x \otimes 1)\left(S_{q}^{*} \otimes u_{q i}^{*}\right) \\
& =\sum_{p, q} S_{p} x S_{q}^{*} \otimes \sum_{i}\left(u_{p i} u_{q i}^{*}\right)=\sum_{p} S_{p} x S_{p}^{*} \otimes 1=\sigma(x) \otimes 1 .
\end{aligned}
$$


To investigate the fixed point algebra, let us consider the following action $\Phi$ of $\mathbf{T}$ on $O_{d}$ given by $\Phi_{z}\left(S_{i}\right)=z S_{i}$; this action commutes with $\Gamma$ :

$$
\Gamma\left(\Phi_{z}\left(S_{i}\right)\right)=z \Gamma\left(S_{i}\right)=z \sum S_{p} \otimes u_{p i}=\sum z S_{p} \otimes u_{p i}=\left(\Phi_{z} \otimes 1\right)\left(\Gamma\left(S_{i}\right)\right) .
$$

Thus if $x$ is a fixed point, then $\Phi_{z} x$ is a fixed point. This means that the fixed point algebra consists of homogeneous parts. The map $\Gamma\left(S_{i}\right)=\sum S_{p} \otimes u_{p i}$ is a map from $H$ to $H \otimes A$. This is a corepresentation of $A$ on $H$. The tensor product is obtained by taking $H^{2}$, this means

$$
S_{i} \otimes S_{j} \rightarrow \sum S_{p} \otimes S_{q} \otimes u_{p i} \otimes u_{q j}, \quad \text { i.e. } S_{i} S_{j} \rightarrow \sum S_{p} S_{q} \otimes u_{p i} u_{q j} .
$$

Thus $\Gamma\left(S_{i} S_{j}\right)=\Gamma\left(S_{i}\right) \Gamma\left(S_{j}\right)$. The trivial corepresentation is given by $\lambda \in \mathbf{C} \rightarrow$ $\lambda \otimes 1 \in \mathbf{C} \otimes A$. Observe that a fixed point $x \in H^{k}$ can be considered as an intertwiner by the following:

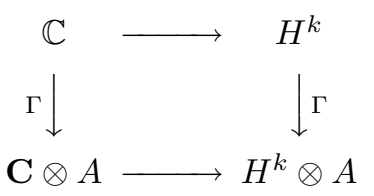

Hence an intertwiner $(H, H)$ is given by a map $T\left(S^{i}\right)=\sum a_{i}^{j} S_{j}$ and satisfies

$$
\begin{gathered}
\Gamma\left(T\left(S_{i}\right)\right)=\sum a_{i}^{j} \Gamma\left(S_{j}\right)=\sum a_{i}^{j} S_{p} \otimes u_{p j}, \\
(T \otimes 1) \Gamma\left(S_{j}\right)=(T \otimes 1) \sum S_{p} \otimes u_{p i}=\sum a_{q}^{p} S_{p} \otimes u_{q i} .
\end{gathered}
$$

This implies that for all $i$ and $p$, we have $\sum_{q} a_{q}^{p} u_{q i}=\sum_{j} a_{q}^{j} u_{p j}$ or $\sum_{i} a_{i}^{p} u_{i q}=$ $\sum_{j} a_{q}^{j} u_{p j}$. Similarly, an intertwining between $H^{2}$ and itself gives relations between the products $u_{i j} u_{p q}$. Consider the quantum $U_{q}(d)$ as in [6]. It is the algebra generated by $u_{p q}$ satisfying the relations

$$
\sum_{k, l} R_{i j}^{k l} u_{k m} u_{l p}=\sum_{k, l} R_{k l}^{m p} u_{i k} u_{j l}
$$

for all $i, j, m, p$ where

$$
\begin{gathered}
R_{i i}^{i i}=q^{-1}, \quad R_{i j}^{j i}=1 \quad \text { for } i \neq j, \\
R_{i j}^{i j}=q^{-1}-q \text { for } i>j, \quad R_{m p}^{i j}=0 \quad \text { otherwise. }
\end{gathered}
$$

These equations mean precisely that $R$ is an intertwiner between $H^{2}$ and $H^{2}$. In this algebra we have the quantum determinant

$$
D=\sum_{\sigma \in S(d)}(-q)^{l(\sigma)} u_{\sigma_{1} 1} \cdots u_{\sigma_{d} d}
$$

This $D$ is in the centre. We have $\Delta(D)=D \otimes D$, where $\Delta\left(u_{i j}\right)=\sum u_{i p} \otimes u_{p j}$. We obtain $U_{q}(d)$ if we require $D$ to be invertible. Then we get a Hopf*-algebra. The antipode can be expressed in terms of the $u$ 's and $D^{-1}$.

Suppose now that $x$ is a fixed point in $O_{d}$ for the action of $U_{q}(d)$. Consider the homomorphism

$$
\gamma: U_{q}(d) \rightarrow U_{q}(d)
$$

defined by

$$
\gamma\left(u_{p q}\right)=0 \text { if } p \neq q ; \quad \gamma\left(u_{p p}\right)=D, \quad \gamma(D)=D^{d} .
$$


Let us recall here also the definition of the compact quantum group $S U_{q}(d)$ as in [13], [8]. It is the algebra generated by elements $\left(u_{p q}\right)$ such that $\left(u_{p q}\right)$ is a unitary matrix and

$$
\sum E\left(k_{0}, k_{1}, \ldots, k_{d}\right) u_{l_{0} k_{0}} u_{l_{1} k_{1}} \cdots u_{l_{d} k_{d}}=E\left(l_{0}, l_{1}, \ldots, l_{d}\right)
$$

where $E\left(k_{0}, k_{1}, \ldots, k_{d}\right)=0$ if two indices are equal and otherwise

$$
E\left(k_{0}, k_{1}, \ldots, k_{d}\right)=(-1)^{I\left(k_{0}, k_{1}, \ldots, k_{d}\right)}
$$

where $I\left(k_{0}, k_{1}, \ldots, k_{d}\right)$ is the number of inverted pairs in the permutation $\left(k_{0}\right.$, $\left.k_{1}, \ldots, k_{d}\right)$. Set $S_{q}=\sum E\left(k_{0}, \ldots, k_{d}\right) S_{k_{0}} \cdots S_{k_{d}}$. This implies

$$
\begin{gathered}
\sum E\left(k_{0}, \ldots, k_{d}\right) \Gamma\left(S_{k_{0}} \cdots S_{k_{d}}\right)=\sum E\left(k_{0}, \ldots, k_{d}\right) S_{l_{0}} \cdots S_{l_{d}} \otimes u_{l_{0} k_{0}} \cdots u_{l_{d} k_{d}} \\
=\sum E\left(e_{0}, \ldots, e_{d}\right) S_{l_{0}} \cdots S_{l_{d}} \otimes 1 .
\end{gathered}
$$

Hence $S_{q}=\sum E\left(k_{0}, \ldots, k_{d}\right) S_{k_{0}} \cdots S_{k_{d}}$ is a fixed point for the coaction $\Gamma$ of $S U_{q}(d)$ on $O_{d}$.

Theorem 5. The fixed point subalgebra $\left(O_{d}\right)^{U_{q}(d)}$ of $O_{d}$ by coaction $\Gamma$ coincides with the fixed point subalgebra $\left(M_{d}^{\infty}\right)^{S U_{q}(d)}$ of the UHF algebra $M_{d}^{\infty}$ by the coaction of $S U_{q}(d)$. In particular

$$
\left(O_{d}\right)^{U_{q}(d)}=\left(M_{d}^{\infty}\right)^{U_{q}(d)}=\left(M_{d}^{\infty}\right)^{S U_{q}(d)}
$$

Proof. Consider the homomorphism $\gamma: U_{q}(d) \rightarrow U_{q}(d)$ defined by $\gamma\left(u_{p q}\right)=0$ if $p \neq q$ and $\gamma\left(u_{p p}\right)=D, \gamma(D)=D^{d}$. We have

$$
\begin{gathered}
(i \otimes \gamma) \Gamma\left(S_{i}\right)=\sum S_{p} \otimes \gamma\left(u_{p i}\right)=S_{i} \otimes D \\
(i \otimes \gamma) \Gamma\left(S_{i}^{*}\right)=S_{i}^{*} \otimes D^{*}
\end{gathered}
$$

Thus $(i \otimes \gamma) \Gamma(y)=y \otimes 1, y \in U H F$. If $x$ is a fixed point for the action of $U_{q}(d)$, then $(i \otimes \gamma) \Gamma(x)=x \otimes \gamma(1)=x \otimes 1$. Since $x$ may be expanded as a linear combination of elements,

$$
S_{i_{1}} \cdots S_{i_{p}} y, z, w S_{i_{1}}^{*} \cdots S_{i_{p}}^{*}
$$

where $y, z, w$ are in the UHF algebra, and

$$
\begin{gathered}
(i \otimes \gamma) \Gamma\left(S_{i_{1}} \cdots S_{i_{n}} y\right)=S_{i_{1}} \cdots S_{i_{n}} y \otimes D^{d} \\
(i \otimes \gamma) \Gamma\left(w S_{i_{1}}^{*} \cdots S_{i_{n}}^{*}\right)=w S_{i_{1}}^{*} \cdots S_{i_{n}}^{*} \otimes D^{-d},
\end{gathered}
$$

we must have that $x \in \mathrm{UHF}$ subalgebra. Next we show that, if $x \in$ UHF subalgebra and is fixed for $S U_{q}(d)$, then it is also fixed for $U_{q}(d)$. Let $x \in \mathrm{UHF}$ and assume $(i \otimes \Phi) \Gamma(x)=x \otimes 1$, where $\Gamma$ is the coaction of $U_{q}(d)$ on $O_{d}$ and $\Phi: U_{q}(d) \rightarrow S U_{q}(d)$, mapping $D$ to 1 . We claim that $\Gamma(x)=x \otimes 1$. Define the quotients of $U_{q}(d)$ with $D=\lambda$, and call these quotients $A_{\lambda}$. Thus $A_{1}=S U_{q}(d)$. Call the quotient map $\pi_{\lambda}: U_{q}(d) \rightarrow A_{\lambda}$. Let the automorphisms $\gamma_{z}: U_{q}(d) \rightarrow U_{q}(d)$ be given by $\gamma_{z}\left(u_{p q}\right)=z u_{p q}$. Then $\gamma_{z}(D)=z^{d} D$. Because $x \in \operatorname{UHF}$ we have $\left(i \otimes \gamma_{z}\right) \Gamma(x)=\Gamma(x)$. Now, $\pi_{\lambda} \gamma_{z}(D)=\pi_{\lambda}\left(z^{d} D\right)=z^{d} \lambda$, so that $\pi_{\lambda} \gamma_{z}=\pi_{z^{d} \lambda}$. If $z^{d} \lambda=1$, then we have

$$
\left(i \otimes \pi_{\lambda}\right) \Gamma(x)=\left(i \otimes \pi_{\lambda} \gamma_{z}\right) \Gamma(x)=\left(i \otimes \pi_{1}\right) \Gamma(x)=(i \otimes \Phi) \Gamma(x)=x \otimes 1 .
$$

This is true for all $\lambda$. Hence $\Gamma(x)=x \otimes 1$.

The statement of our main result is as follows. 
Corollary 6. The fixed point subalgebra $\left(O_{d}\right)^{U_{q}(d)}$ is generated as a $C^{*}$-algebra by $\theta(g), g \in B_{\infty}$.

Proof. Consider an element $T$ in $\left(O_{d}\right)^{U_{q}(d)}$ : it is then a fixed point under the coaction of $U_{q}(d)$ on $O_{d}$. Note that any element in the fixed point subalgebra $\left(M_{d}^{\infty}\right)^{U_{q}(d)}$ or, equivalently in $\left(M_{d}^{\infty}\right)^{S U_{q}(d)}$ by Theorem 5 , is an intertwiner of $\left(H^{k}, H^{k}\right)$.

Let $T \in\left(H^{k}, H^{k}\right)$ for some $1 \leq k \leq d$. Let $S_{q}$ be as defined before, and note that $S_{q} \in\left(i, H^{d}\right)$. For any $T \in\left(H^{k}, H^{k}\right)$ form $T_{k}^{\prime}=T \otimes 1_{H^{d-k}}, 1 \leq k \leq d, T_{k}^{\prime}$ is an intertwiner of $\left(H^{d}, H^{d}\right)$.

Set $\widetilde{T}_{k}=T_{k}^{\prime} S_{q}$. Let $\widetilde{T}_{k}$ and $\widetilde{T}_{k}^{\prime}$ be any two such intertwiners. It follows that $\widetilde{T}_{k}^{*} \widetilde{T}_{k} \in(\mathbf{C}, \mathbf{C})$, so it is a scalar. As in [3] we can define the scalar product by:

$$
\widetilde{T}_{k}^{*} \widetilde{T}_{k}^{\prime}=\left\langle\widetilde{T}_{k}, \widetilde{T}_{k}^{\prime}\right\rangle 1 .
$$

This will give the structure of Hilbert space to the set of intertwiners.

An orthonormal basis can be described as follows. Let $G$ be a graph whose vertices correspond to the labels, i.e. configurations of tensor powers appearing in the specific intertwiner. We denote the generic source vertex by $\alpha \equiv \alpha_{0}$ and by $\beta \equiv \alpha_{n}$ the generic "sink".

Let $e_{i}$ be the directed edge that goes from the label $\alpha_{i-1}$ to the label $\alpha_{i}, i=$ $1, \ldots, n$. Each of these directed edges is an intertwiner. We denote it by $T_{e_{i}}=$ $T_{\alpha_{i-1}, \alpha_{i}}$. It is the intertwiner that represents the $i$ th edge $e_{i}$ from $\alpha_{i-1}$ to $\alpha_{i}$, i.e. $e_{i}=\left(\alpha_{i-1}, \alpha_{i}\right)$. We say, as in [7], that a path $\xi$ of length $n$ from $\alpha$ to $\beta$ is a sequence $\xi=\left(e_{1}, \ldots, e_{n}\right)$ of edges, where $e_{1}$ starts at $\alpha_{0}$, and the endpoint of each $e_{h}$, for $h=1, \ldots, n-1$, is the initial point of $e_{h+1}$, and $e_{n}$ ends at $\alpha_{n}$. We denote the set of these paths by $P a t h_{\alpha, \beta}^{(n)}$. In our situation the number of terms in labels (or configuration) $\alpha$ and $\beta$ will be the same, since the intertwiners are between objects with equal tensor power. Denote by $H_{\alpha, \beta}^{(n)}$ the Hilbert space with inner product given by (3), and let $n$ denote the minimal number of steps (or edges) needed to go from $\alpha$ to $\beta$. A convenient orthonormal basis in $H_{\alpha, \beta}^{(n)}$ is the set of products of the distinguished basis elements in the spaces $H_{\alpha, \beta}^{(i)}$. Thus the intertwiners $T(\xi)=$ $T_{e_{1}} \cdots T_{e_{n}}$ with $\xi \in$ Paths $_{\alpha, \beta}^{(n)}$ are an orthonormal basis. Observe that to any intertwiner $A \in\left(H^{k}, H^{k}\right)$, there is associated a permutation which we denote by $p_{A} \in S(k)$, where $S(k)$ represents the symmetric group on $k$ letters, which allows us to go from the labels $\alpha$ to the labels $\beta$. Every $p_{A}$ can be decomposed as products of at most $k$ disjoint cycles, i.e. $p_{A}=\sigma_{i_{1}} \cdots \sigma_{i_{k}}$. Hence for every $\sigma_{i_{j}}$ there exists an edge $e_{j}$ connecting the top configuration to the one immediately below. Thus to each $A$ there is associated a path, say $\xi_{A}$. The composition law for paths is the following: $\xi \circ \xi^{\prime}$ is possible if the bottom number of labels of $\xi$ and the top number of labels of $\xi^{\prime}$ agree. Observe that we have the following multiplication rule for the operator $A(\xi) A\left(\xi^{\prime}\right)^{*}$ :

$$
A(\xi) A\left(\xi^{\prime}\right)^{*} A(\eta) A\left(\eta^{\prime}\right)^{*}=\delta_{\xi^{\prime} \eta} A(\xi) A\left(\eta^{\prime}\right)^{*}
$$

which is the analogue of the discrete Witten's product for strings [10]. To actually compute the expression of any $A(\xi) \in\left(H^{k}, H^{k}\right)$ let us proceed as follows. Consider $\xi$ to be an elementary path. Hence

$$
\left\langle A(\xi) \widetilde{T}_{k}\left(\xi^{\prime}\right), \widetilde{T}_{k}\left(\eta^{\prime}\right)\right\rangle=\widetilde{T}_{k}\left(\eta^{\prime}\right)^{*} A(\xi) \widetilde{T}_{k}\left(\xi^{\prime}\right) .
$$


This means project $A(\xi)$ on paths given by $S_{q}$, i.e. $\widetilde{T}_{k}\left(\eta^{\prime}\right)^{*} A(\xi) \widetilde{T}_{k}\left(\xi^{\prime}\right)=c\left(\xi^{\prime}, \eta\right) \delta_{\xi^{\prime}, \eta}$. In the above formula, we denote by $c\left(\xi^{\prime}, \eta\right)$ the scalar associated to the operator on the edges of $\xi^{\prime}, \eta$. Denote the operator and its values by the same symbol $c\left(\xi^{\prime}, \eta\right)$ since it can be viewed as an operator on paths. We want to prove that it satisfies the Yang-Baxter equation. We may think of $\xi^{\prime}, \eta$ as elementary paths for simplicity, i.e. paths that move only a pair of indices, the rest of the indices acting just like the identity. Thus $c\left(\xi^{\prime}, \xi^{\prime}\right)\left(h_{1} \otimes h_{2}\right)=\sum s_{k} h_{1} \otimes t_{i} h_{2}, s_{k}, t_{i} \in \mathbf{C}$, associated to $\xi^{\prime}$. We need then to prove

$$
c_{23}\left(\xi^{\prime}, \xi^{\prime}\right) c_{13}\left(\xi^{\prime}, \xi^{\prime}\right) c_{12}\left(\xi^{\prime}, \xi^{\prime}\right)=c_{12}\left(\xi^{\prime}, \xi^{\prime}\right) c_{13}\left(\xi^{\prime}, \xi^{\prime}\right) c_{23}\left(\xi^{\prime}, \xi^{\prime}\right)
$$

where the lower indices denote the position of $c\left(\xi^{\prime}, \xi^{\prime}\right)$ on a string of a 3-tensor. To prove (5) we need to check that the left hand side and the right hand side agree. Hence, calculating the left hand side we have

$$
c_{23}\left(\xi^{\prime}, \xi^{\prime}\right) c_{13}\left(\xi^{\prime}, \xi^{\prime}\right) c_{12}\left(\xi^{\prime}, \xi\right)=\sum_{\substack{k, k^{\prime} \\ r, s, i^{\prime}, i}} s_{k^{\prime}} s_{k} h_{1} \otimes s_{r} t_{i} h_{2} \otimes t_{s} t_{i^{\prime}} h_{3} .
$$

However, on the right hand side

$$
c_{12}\left(\xi^{\prime}, \xi^{\prime}\right) c_{13}\left(\xi^{\prime}, \xi\right) c_{23}\left(\xi^{\prime}, \xi^{\prime}\right)=\sum_{\substack{k, k^{\prime} \\ r, s, i^{\prime}, i}} s_{r} s_{k^{\prime}} h_{1} \otimes t_{s} s_{k} h_{2} \otimes t_{i^{\prime}} t_{i} h_{3} .
$$

Looking at the two expressions, we observe that the only difference is in the middle part. Since the cycles of the permutations are disjoint no matter which edge we apply first, we get the same result. Hence $c\left(\xi^{\prime}, \xi^{\prime}\right)$ is a Yang-Baxter operator. Hence it gives an element of the braid group.

Therefore the space of intertwiners carries a representation of the braid group given by the Yang-Baxter operator $c\left(\xi^{\prime}, \xi^{\prime}\right)$. By Theorem VIII.6.4 of [5] we have $c\left(\xi^{\prime}, \xi^{\prime}\right)=c_{H, H}=\tau \circ R$, with $R$ the $R$-matrix associated to $U_{q}(d)$, when $\xi^{\prime}=\eta$ is the path from $H \otimes H \rightarrow H \otimes H$. It follows that to any intertwiner $A \in\left(H^{k}, H^{k}\right)$ we can associate a path $\xi$ which corresponds to a braid group generator $g$ with the permutation $p=p(g)$. From [9] and [13] it is already known that the algebra of the linear intertwiners $\left(H^{k}, H^{k}\right)$ under the coaction of quantum $S U(d)$ is generated by a representation of the braid group $B_{\infty}$.

Then $A(\xi)$ can be expressed as a combination of the braid group representation $\theta(g)$ and by using the relations (1) on the generators of the Cuntz algebra, we can express

$$
\theta(g)=\sum_{i_{1} \cdots i_{k}} c_{i_{1} \cdots i_{k}}^{p\left(i_{k}\right), \ldots, p\left(i_{1}\right)} S_{i_{1}} \cdots S_{i_{k}} S_{p\left(i_{k}\right)}^{*} \cdots S_{p\left(i_{1}\right)}^{*}
$$

where we denote by $c_{i_{1} \cdots i_{k}}^{p\left(i_{k}\right), \cdots, p\left(i_{1}\right)}$ the matrix elements of the operator $c\left(\xi^{\prime}, \xi^{\prime}\right)$ associated to the path corresponding to the braid group element $g$. By using Lemma 6 of [9] we conclude that $\left(O_{d}\right)^{U_{q}(d)}=C^{*}(\theta(g))$.

Lemma 7. $\left(O_{d}\right)^{S U_{q}(d)}$ is the smallest $\sigma$-stable $C^{*}$-subalgebra of $O_{d}$ containing $\theta(g)$, $g \in B_{\infty}$, and $S_{q}$.

Proof. We want to prove that every element in the fixed point subalgebra $\left(O_{d}\right)^{S U_{q}(d)}$ is obtained from elements of $\left(O_{d}\right)^{U_{q}(d)}$ plus the additional element $S_{q}$. Let $X \in$ $\left(O_{d}\right)^{S U_{q}(d)}$ so that $\Gamma(X)=X \otimes 1$. Now by Lemma 5 and Lemma 6 of [9] it is enough to prove for $x \in P_{d} \cap\left(O_{d}\right)^{S U_{q}(d)}$ where $P_{d}$ is the dense *-subalgebra of $O_{d}$ 
generated by $S_{1}, \ldots, S_{d}$. Thus by [1] we can write any $x$ as a linear combination of elements $S_{i_{1}} \cdots S_{i_{p}} y, z, w S_{i_{1}}^{*} \cdots S_{i_{p}}^{*}$ where $y, z, w \in$ UHF subalgebra of $O_{d}$.

We need to show that if $X \in\left(O_{d}\right)^{U_{q}(d)}$, then $X$ contains a part in $\left(O_{d}\right)^{U_{q}(d)}$ and a part in $\left(O_{d}\right)^{S U_{q}(d)}$ which includes $S_{q}$. Let us proceed as follows. We need to have grading. The grading in $\left(O_{d}\right)^{S U_{q}(d)}$ is defined as follows. Let the automorphism $\gamma_{z}: U_{q}(d) \rightarrow U_{q}(d)$ be given by $\gamma_{z}\left(u_{p q}\right)=z^{k} u_{p q}$; then $\gamma_{z}(D)=z^{d k} D$, $z \in \mathbf{T}$. Let $\Gamma_{z}(X)=\left(\right.$ id $\left.\otimes \gamma_{z}\right) \Gamma(X)$. Then we define $\left(\left(O_{d}\right)^{S U_{q}(d)}\right)^{k}$, all $X$ such that $\left(\mathrm{id} \otimes \gamma_{z}\right) \Gamma(X)=z^{k} \Gamma(X)$, i.e. equivalently

$$
\left(\left(O_{d}\right)^{S U_{q}(d)}\right)^{k}=\left\{X \in\left(O_{d}\right)^{S U_{q}(d)}: \Gamma_{z}(X)=z^{k} X \otimes 1\right\} .
$$

Let $X \in\left(\left(O_{d}\right)^{S U_{q}(d)}\right)^{0}$ where $k=0$, and $\Gamma_{z}(X)=\left(\operatorname{id} \otimes \gamma_{z}\right) \Gamma(X)$. We have seen that $\left(\operatorname{id} \otimes \gamma_{z}\right) \Gamma(X)=\Gamma(x)$ if and only if $X \in(U H F)^{U_{q}(d)}=\left(O_{d}\right)^{U_{q}(d)}$. Thus the zero part of $\left(\left(O_{d}\right)^{S U_{q}(d)}\right)^{0}=\left(O_{d}\right)^{U_{q}(d)}$. Now, observe that $\left(\left(O_{d}\right)^{S U_{q}(d)}\right)^{k}=0$, unless $k=0(\bmod d)$. Since $u_{i j} \in S U_{q}(d)$ and the quantum determinant $D=1$ in $S U_{q}(d)$, it follows that $z^{d}=1$. We claim that $\left(X S_{q}^{n *}\right) \in\left(\left(O_{d}\right)^{S U_{q}(d)}\right)^{0}$ where $X \in\left(\left(O_{d}\right)^{S U_{q}(d)}\right)^{n d}$. Observe that

$$
\begin{aligned}
\Gamma_{z}\left(X S_{q}^{n *}\right) & =\Gamma_{z}(X) \Gamma_{z}\left(S_{q}^{n *}\right)=\left(z^{n d} X \otimes 1\right)\left(z^{-n d} S_{q}^{n *} \otimes 1\right) \\
& =z^{n d-n d} X S_{q}^{n *} \otimes 1=\Gamma\left(X S_{q}^{n *}\right)
\end{aligned}
$$

which implies $X S_{q}^{n *} \in\left(\left(O_{d}\right)^{S U_{q}(d)}\right)^{0}$. Then it follows $X S_{q}^{n *} \in\left(\left(O_{d}\right)^{S U_{q}(d)}\right)^{0}=$ $\left(O_{d}\right)^{U_{q}(d)}$. Since $X S_{q}^{n *} \in\left(O_{d}\right)^{U_{q}(d)}$ we have $\Gamma\left(X S_{q}^{n *}\right)=\left(\operatorname{id} \otimes \gamma_{z}\right) \Gamma\left(X S_{q}^{n *}\right)$ which is true for every $z$. If $X \in\left(\left(O_{d}\right)^{S U_{q}(d)}\right)^{n d}$ set $X=k(q)^{n} X S_{q}^{n *} S_{q}^{n}$ where $S_{q}^{n *} S_{q}^{n}=$ $k(q)^{-n}$. Then

$$
\Gamma_{z}\left(k(q)^{n} X S_{q}^{n *}\right)=k(q)^{n} X S_{q}^{n *} \otimes 1=\Gamma\left(k(q)^{n} X S_{q}^{n *}\right) .
$$

This implies that $T=k(q)^{n} X S_{q}^{n *} \in\left(O_{d}\right)^{U_{q}(d)}$. Thus every $X \in\left(O_{d}\right)^{S U_{q}(d)}$ consists of parts containing $\left(O_{d}\right)^{U_{q}(d)}$ and $S_{q}$. As $\left(O_{d}\right)^{S U_{q}(d)}$ is $\sigma$-stable, the result follows from Corollary 6.

\section{ACKNOWLEDGMENT}

The author expresses her thanks to Professor G. Elliott for the support received during her stay at the Fields Institute, and to Professors A. Van Daele and P. E. T. Jorgensen for useful discussions on the subject.

\section{REFERENCES}

[1] J. Cuntz, (1977), Simple $C^{*}$-algebras generated by isometries, Comm. Math. Phys. 57, p. 173-185. MR 57:7189

[2] J. Cuntz, Regular actions of Hopf algebras on the $C^{*}$-algebra generated by a Hilbert space, preprint. MR 94m:46104

[3] S. Doplicher, J. E. Roberts, (1987), Duals of compact Lie groups realized in the Cuntz algebras, J. Functional Analysis, 74, 90-120. MR 89a:22011

[4] K. Fredenhagen, K. H. Rehren, and B. Schroer, (1989), Superselection sectors with braid group statistics and exchange algebras I, Comm. Math. Phys. 125, p. 201-226. MR 91c:81047

[5] Kassel, (1994), Quantum Groups, Springer Verlag.

[6] H. T. Koelink, (1991), On *-representations of the Hopf $*$-algebra associated with the quantum group $U_{q}(n)$, Compositio Mathematica, 77, 199-231. MR 92b:16080

[7] A. Ocneanu, (1972), Path algebras, A.M.S. meeting in Santa Cruz.

[8] A. Van Daele, (1993), Dual pairs of Hopf *-algebras, Bull. London Math. Soc., 25, 209-230. MR 94c: 16053 
[9] Y. Konishi, M. Nagisa, and Y. Watatani, (1992), Some remarks on actions of compact matrix quantum groups on $C^{*}$-algebras, Pacific J. of Mathematics, 153, p. 119-127. MR 93c:46121

[10] E. Witten, (1986), Noncommutative geometry and string field theory, Nuclear Physics B 268, p. 253-294.

[11] S. L. Woronowicz, (1987a), Compact matrix pseudogroups, Comm. Math. Phys. 111, 613-665. MR 88m:46079

[12] S. L. Woronowicz, (1987b), Twisted $S U(2)$ group, An example of a noncommutative differential calculus, Publ. RIMS 23, 117-181. MR 88h:46130

[13] S. L. Woronowicz, (1988), Tannaka-Krein duality for compact matrix pseudogroups, twisted $S U(N)$ groups, Inventiones Math., 93, p. 35-76. MR 90e:22033

[14] S. L. Woronowicz, Compact quantum groups, preprint March 1992.

The Fields Institute, 185 Columbia St. West, Waterloo, Ontario, Canada N2L 5Z5

Current address: School of Mathematics, University of Leeds, LS2 9JT United Kingdom

E-mail address: paolucci@amsta.leeds.ac.uk 